\title{
Amplifying stationary quantum discord and entanglement between a superconducting qubit and a data bus by time-dependent electromagnetic field
}

\author{
QIAN Yi, ZHANG YeQi \& XU JingBo* \\ Zhejiang Institute of Modern Physics and Physics Department, Zhejiang University, Hangzhou 310027, China
}

Received November 3, 2011; accepted January 12, 2012

\begin{abstract}
We study the dynamics of quantum discord and entanglement between a superconducting qubit and a data bus, which is driven by a controllable time-dependent electromagnetic field, in the presence of phase decoherence and find that the quantum discord and entanglement remain at a stationary non-zero value for long time evolution. It is shown that the amount of stationary quantum discord and entanglement can be enhanced by applying the time-dependent electromagnetic field.
\end{abstract}

stationary quantum discord, stationary entanglement, time-dependent electromagnetic field

Citation: Qian Y, Zhang Y Q, Xu J B. Amplifying stationary quantum discord and entanglement between a superconducting qubit and a data bus by time-dependent electromagnetic field. Chin Sci Bull, 2012, 57: 1637-1642, doi: 10.1007/s11434-012-5112-4

Quantum entanglement plays a crucial role in quantum information and computation. It is a special quantum correlation and has been recognized as an essential resource in quantum information processing [1]. The experimental demonstrations of two-particle entanglement and multi-particle entanglement in the cavity quantum electrodynamics have been reported [2,3] and some applications about the entanglement or the nonclassical correlations have also been realized in recent experiments [4-6]. However, some recent studies show that there are other kinds of quantum correlations that can offer support for the quantum tasks. It has been found both theoretically [7-9] and experimentally [10,11] that other nonclassical correlation, namely, quantum discord [12] can be responsible for computational speedup for certain quantum tasks [13-19] compared to their classical counterparts. Quantum discord, introduced by Ollivier and Zurek [12], is a general measure of quantum correlation and defined as the difference between the quantum mutual information and the classical correlation. The main difference between the quantum discord and entanglement is the fact that the quantum discord is nonzero even for separate mixed states. Therefore,

*Corresponding author (email: xujb@zju.edu.cn) the quantum discord may be regarded as a more general and fundamental resource for the quantum information processing. Recently, much attention has been focused on the study of the dynamics of quantum discord for some open systems $[15,20,21]$. It has been shown that the quantum discord can be completely unaffected by the certain decoherence environment during an initial time interval [22] and this phenomenon has been verified by the recent experiment [23].

On the other hand, the superconduting qubits have been considered as possible candidates for quantum information processing and attracted much attention in recent years [2431]. It has been experimentally demonstrated that the superconducting qubits possess macroscopic quantum coherence and can be used to construct the conditional two-qubit gate. It is necessary to explore possibility for scaling up to many qubits in order to perform the complex quantum information task. Several schemes have been proposed to selectively couple any pair of qubits with a common data bus [26-29]. In [30], Liu et al. proposed a method to use a controllable timedependent electromagnetic field to couple a superconducting qubit and data bus, where quantum information can be transferred from one qubit to another.

In this paper, we investigate the dynamics of quantum 
discord and entanglement between a data bus and a superconducting qubit, which is driven by a controllable timedependent electromagnetic field (TDEF), in the presence of phase decoherence and find that the quantum discord and entanglement remain at a stationary non-zero value for long time evolution. This means that the stationary quantum discord and entanglement can arise in the interaction of the superconducting qubit and data bus as the time approach to infinite. We also examine the influence of the TDEF on the dynamics of quantum discord and entanglement between the superconducting qubit and data bus and show that the amount of stationary quantum discord and entanglement can be increased by applying the TDEF.

\section{Time evolution of superconducting qubit system driven by an electromagnetic field with phase decoherence}

We now consider a superconducting flux qubit interacting with a data bus and a controllable TDEF. The data bus can be realized simply by an LC circuit and the interaction between a single superconducting flux qubit and a LC circuit has been realized experimentally. The Hamiltonian of the system can be described as $[30,31]$ (note that we have set $\hbar=1$ in this paper)

$$
\begin{gathered}
H=H_{0}+H_{F}, \\
H_{0}=\frac{\omega_{q}}{2} \sigma_{z}+\frac{\omega^{2} L Q^{2}}{2}+\frac{\omega^{2} C \widetilde{\phi}^{2}}{2} \\
+\left[\chi\left(\sqrt{\frac{\omega C}{2}} \widetilde{\phi}-i \sqrt{\frac{\omega L}{2}} Q\right) \sigma_{+}+H . c .\right], \\
H_{F}=\lambda\left(\mathrm{e}^{-i \omega_{e} t} \sigma_{+}+\mathrm{e}^{i \omega_{e} t} \sigma_{-}\right),
\end{gathered}
$$

where H.c. means Hermitian conjugate, $\omega=1 / \sqrt{L C}$ (with the the capacitor $C$ and the self-inductance $L$ ) and $\omega_{q}=$ $\frac{1}{2} \sqrt{I^{2}\left(\Phi_{e}-\frac{\Phi_{0}}{2}\right)^{2}+T_{R L}^{2}}$ are the frequency of data bus and qubit, respectively. Here, $\Phi_{e}$ is the externally applied flux, $\Phi_{0}$ is the flux quantum, $I$ is the qubit loop-current and the parameter $T_{R L}$ denotes the tunnel coupling between the two potential wells of the qubit. The $H_{F}$ denotes a controllable TDEF and $\omega_{e}$ is the frequency of the TDEF, $\lambda$ is the coupling constants of the interactions of qubit with the TDEF. The magnetic flux $\widetilde{\phi}$ through the LC circuit and the charge $Q$ operators obey the commutation relation $[Q, \widetilde{\phi}]=i$. The operators $\sigma_{z}$ and $\sigma_{ \pm}$are defined by $\sigma_{z}=|e\rangle\langle e|-| g\rangle\left\langle g\left|, \sigma_{+}=\right| e\right\rangle\langle g|$, $\sigma_{-}=|g\rangle\langle e|$, where $|e\rangle$ and $|g\rangle$ are the exited state and ground state of the qubit. $\chi$ is the coupling constant between the qubit and the data bus and can be written as $\chi=M \sqrt{\frac{\omega}{2 L}}\langle e|I| g\rangle$ (in this paper we have set $\chi$ to be a real number so that $\chi=\chi^{*}$ ).

In the rotating reference frame, through an unitary trans- formation $U=U_{2} U_{1}$, where

$$
\begin{aligned}
U_{1} & =\exp \left(-\frac{i \omega_{e} t}{2} \sigma_{z}\right), \\
U_{2} & =\exp \left[-\frac{i \omega_{e} t}{2}\left(\frac{\omega_{q}-\omega_{e}}{2 \delta} \sigma_{z}+\frac{\lambda}{\delta} \sigma_{x}\right)\right],
\end{aligned}
$$

the Hamiltonian of eq. (2) is transformed to [30]

$$
\begin{aligned}
H^{\prime}= & \frac{\omega L Q^{2}}{2}+\frac{\omega C \widetilde{\phi}^{2}}{2}+\frac{\delta+\omega_{e}}{2}\left(\frac{\omega_{q}-\omega_{e}}{\delta} \sigma_{z}+\frac{2 \lambda}{\delta} \sigma_{x}\right) \\
& +\left[\frac{\chi\left(\delta+\omega_{q}-\omega_{e}\right)}{2 \delta}\left(\sqrt{\frac{\omega C}{2}} \widetilde{\phi}-i \sqrt{\frac{\omega L}{2}} Q\right)\right. \\
& \left.\otimes\left(\frac{\omega_{q}-\omega_{e}}{2 \delta} \sigma_{x}+\frac{i}{2} \sigma_{y}-\frac{\lambda}{\delta} \sigma_{z}\right)+\text { H.c. }\right],
\end{aligned}
$$

where $\sigma_{x}=|e\rangle\langle g|+| e\rangle\langle g|, \sigma_{y}=-i(|e\rangle\langle g|-| e\rangle\langle g|)$ and $\delta=\sqrt{\left(\omega_{q}-\omega_{e}\right)^{2}+4 \lambda^{2}}$.

We consider the pure phase decoherence mechanism only. In this situation, the master equation governing the time evolution for the quantum system with phase decoherence is given by [32]

$$
\frac{\mathrm{d} \rho}{\mathrm{d} t}=-i\left[H^{\prime}, \rho\right]-\frac{\gamma}{2}\left[H^{\prime},\left[H^{\prime}, \rho\right]\right],
$$

where $\gamma$ is the phase decoherence coefficient. Eq. (6) reduces to the ordinary von Neuman equation when $\gamma \rightarrow 0$. The formal solution of the master equation can be expressed as [32]

$$
\rho(t)=\sum_{k=0}^{\infty} \frac{(\gamma t)^{k}}{k !} M^{k}(t) \rho(0) M^{\dagger k}(t),
$$

where $\rho(0)$ is the initial density operator of the system and $M^{k}(t)$ is defined by

$$
M^{k}(t)=H^{\prime k} \exp \left(-i H^{\prime} t\right) \exp \left(-\frac{\gamma t}{2} H^{\prime 2}\right) .
$$

With the help of the $S U(2)$ dynamical algebraic structure, we can rewrite $H^{\prime}$ as [33]

$H^{\prime}=\Delta S_{0}+\omega\left(K-\frac{1}{2}\right)+\frac{\sqrt{K}}{2}\left(1+\frac{\omega_{q}-\omega_{e}}{\delta}\right) \chi\left(S_{+}+S_{-}\right)$,

where $\Delta=\delta+\omega_{e}-\omega, K=\frac{1}{2}\left(\frac{\omega_{q}-\omega_{e}}{\delta} \sigma_{z}+\frac{2 \lambda}{\delta}\right)+\frac{1}{2}\left(L \widetilde{\phi}^{2}+L Q^{2}\right) \omega$ is a constant of motion which commutes with the Hamiltonian $H^{\prime}$ and the operators

$$
\begin{aligned}
& S_{+}=\sqrt{\frac{1}{K}}\left(\sqrt{\frac{\omega C}{2}} \widetilde{\phi}-i \sqrt{\frac{\omega L}{2}} Q\right)\left(\frac{\omega_{q}-\omega_{e}}{2 \delta} \sigma_{x}+\frac{i}{2} \sigma_{y}-\frac{\lambda}{\delta} \sigma_{z}\right), \\
& S_{-}=\sqrt{\frac{1}{K}}\left(\sqrt{\frac{\omega C}{2}} \widetilde{\phi}+i \sqrt{\frac{\omega L}{2}} Q\right)\left(\frac{\omega_{q}-\omega_{e}}{2 \delta} \sigma_{x}-\frac{i}{2} \sigma_{y}-\frac{\lambda}{\delta} \sigma_{z}\right) \\
& S_{0}=\frac{1}{2}\left(\frac{\omega_{q}-\omega_{e}}{\delta} \sigma_{z}+\frac{2 \lambda}{\delta} \sigma_{x}\right)
\end{aligned}
$$

satisfy the following commutation relations:

$$
\left[S_{0}, S_{ \pm}\right]= \pm S_{ \pm},\left[S_{+}, S_{-}\right]=2 S_{0},
$$

which means that the operators $S_{i}(i=0, \pm)$ are the generators of the $S U(2)$ algebra. 
By means of the $S U(2)$ dynamical algebraic structure, we can diagonalize the Hamiltonian in eq. (9) and obtain the explicit expression for the operator $M^{k}(t)$ in eq. (8)

$$
\begin{aligned}
M^{k}(t)= & \frac{1}{2}\left[f_{+}(K)^{k} \exp \left(-i f_{+}(K) t\right) \exp \left(-\frac{\gamma f_{+}(K)^{2} t}{2}\right)\right. \\
& \left.+f_{-}(K)^{k} \exp \left(-i f_{-}(K) t\right) \exp \left(-\frac{\gamma f_{-}(K)^{2} t}{2}\right)\right] \\
& +\left[f_{+}(K)^{k} \exp \left(-i f_{+}(K) t\right) \exp \left(-\frac{\gamma f_{+}(K)^{2} t}{2}\right)\right. \\
& \left.-f_{-}(K)^{k} \exp \left(-i f_{-}(K) t\right) \exp \left(-\frac{\gamma f_{-}(K)^{2} t}{2}\right)\right] \\
& \otimes\left[\frac{\Delta}{4 \Omega_{K}}\left(2 \cos \theta \sigma_{z}+\sin \theta \sigma_{x}\right)\right. \\
& +\frac{\chi \cos ^{2} \frac{\theta}{2}}{2 \Omega_{K}}\left(a\left(-\sin \theta \sigma_{z}+\cos \theta \sigma_{x}+i \sigma_{y}\right)\right. \\
& \left.\left.+a^{\dagger}\left(-\sin \theta \sigma_{z}+\cos \theta \sigma_{x}-i \sigma_{y}\right)\right)\right]
\end{aligned}
$$

where

$$
\begin{aligned}
& \Omega_{K}=\sqrt{\Delta^{2}+4 K\left(\chi \cos ^{2} \frac{\theta}{2}\right)^{2}}, \\
& f_{ \pm}(K)=\omega\left(K-\frac{1}{2}\right) \pm \frac{1}{2} \Omega_{K}, \\
& \theta=\arctan \left(\frac{2 \lambda}{\omega_{q}-\omega_{e}}\right)
\end{aligned}
$$

and the operators

$$
\begin{aligned}
& a=\sqrt{\frac{\omega C}{2}} \widetilde{\phi}-i \sqrt{\frac{\omega L}{2}} Q, \\
& a^{\dagger}=\sqrt{\frac{\omega C}{2}} \widetilde{\phi}+i \sqrt{\frac{\omega L}{2}} Q,
\end{aligned}
$$

satisfy the commutation relation $\left[a, a^{\dagger}\right]=1$.

We assume that the superconducting qubit is initially prepared in the dressed state $|+\rangle=\cos \frac{\theta}{2}|e\rangle+\sin \frac{\theta}{2}|g\rangle$ and the data bus is initially prepared in the fock state $|n\rangle$. Then, the initial density operator of the whole system reads

$$
\rho(0)=|+\rangle|n\rangle\langle+|\langle n| \text {. }
$$

By making use of the eqs. (7), (12), (14), (15), we obtain the density matrix of the system at time $t$ by means of the standard basis $|-\rangle|n\rangle,|-\rangle|n+1\rangle,|+\rangle|n\rangle,|+\rangle|n+1\rangle$ (where $\left.|+\rangle=\cos \frac{\theta}{2}|e\rangle+\sin \frac{\theta}{2}|g\rangle,|-\rangle=-\sin \frac{\theta}{2}|e\rangle+\cos \frac{\theta}{2}|g\rangle\right)$, as follows:

$$
\rho(t)=\left(\begin{array}{cccc}
\rho_{11} & 0 & 0 & \rho_{14} \\
0 & \rho_{22} & \rho_{23} & 0 \\
0 & \rho_{32} & \rho_{33} & 0 \\
\rho_{41} & 0 & 0 & \rho_{44}
\end{array}\right),
$$

where

$\rho_{11}=\rho_{44}=\rho_{14}=\rho_{41}=0$,

$$
\begin{aligned}
\rho_{22}= & \frac{2\left(\chi \cos ^{2} \frac{\theta}{2}\right)^{2}(n+1)}{\Omega_{n+1}^{2}}\left[1-\cos \left(\Omega_{n+1} t\right) \exp \left(-\frac{\gamma \Omega_{n+1}^{2}}{2} t\right)\right], \\
\rho_{33}= & \frac{1}{2}\left[1+\frac{\Delta^{2}}{\Omega_{n+1}^{2}}+\left(1-\frac{\Delta^{2}}{\Omega_{n+1}^{2}}\right) \cos \left(\Omega_{n+1} t\right) \exp \left(-\frac{\gamma \Omega_{n+1}^{2}}{2} t\right)\right], \\
\rho_{32}= & \left(\rho_{23}\right)^{*}=\frac{\chi \cos ^{2} \frac{\theta}{2} \sqrt{n+1}}{\Omega_{n+1}}\left[\frac{\Delta}{\Omega_{n+1}}+\left(i \sin \left(\Omega_{n+1} t\right)\right.\right. \\
& \left.\left.-\frac{\Delta}{\Omega_{n+1}} \cos \left(\Omega_{n+1} t\right)\right) \exp \left(-\frac{\gamma \Omega_{n+1}^{2}}{2} t\right)\right] .
\end{aligned}
$$

It is obvious that the density matrix $\rho(t)$ belongs to the class of $X$ structure states. The advantage of $X$ structure states is that, not only it is easy to calculate the quantum discord and entanglement, but it keeps the $X$ structure unchanged during time evolution as well [34].

\section{The dynamics of quantum discord and en- tanglement for the superconducting qubit sys- tem driven by an electromagnetic field with phase decoherence}

In this section, we study the the influence of phase decoherence and TDEF on the dynamics of quantum discord and entanglement of a superconducting qubit interacting with a data bus in the presence of phase decoherence. The definition of quantum discord is based on quantum mutual information which contains both classical and quantum correlations. For a bipartite system $\rho^{A B}$, its total correlations can be measured by their quantum mutual information [12]:

$$
\mathcal{I}\left(\rho^{A B}\right)=S\left(\rho^{A}\right)+S\left(\rho^{B}\right)-S\left(\rho^{A B}\right),
$$

where $S(\rho)=-\operatorname{Tr}\left(\rho \log _{2} \rho\right)$ is the von Neumann entropy, and $\rho^{A}$ and $\rho^{B}$ denote the reduced density matrices of parts $A$ and $B$, respectively. The quantum discord is defined as the difference between the quantum mutual information and the classical correlation [12]:

$$
\mathcal{D}\left(\rho^{A B}\right)=I\left(\rho^{A B}\right)-C\left(\rho^{A B}\right),
$$

where $C\left(\rho^{A B}\right)$ is the classical correlation which depends on the maximal information obtained with measurement on one of the subsystems and can be expressed as [34]

$$
C\left(\rho^{A B}\right)=\max _{\left\{B_{k}\right\}}\left[S\left(\rho^{A}\right)-S\left(\rho^{A B} \mid\left\{B_{k}\right\}\right)\right],
$$

where $\left\{B_{k}\right\}$ is a complete set of projectors preformed on subsystem $B$ locally, $S\left(\rho^{A B} \mid\left\{B_{k}\right\}\right)=\sum_{k} p_{k} S\left(\rho_{k}\right)$ is the quantum conditional entropy, $\rho_{k}=1 / p_{k}\left(I \otimes B_{k}\right) \rho^{A B}\left(I \otimes B_{k}\right)$ is the conditional density operator and $p_{k}=\operatorname{tr}_{(A B)}\left[\left(I \otimes B_{k}\right) \rho^{A B}\left(I \otimes B_{k}\right)\right]$ is the probability.

The eigenvalues of the density matrix $\rho(t)$ for the qubitdata bus system with TDEF applied under the phase decoher- 
ence can be derived as

$$
\begin{aligned}
& \lambda_{1,2}=\frac{1}{2}\left[\rho_{11}+\rho_{44} \pm \sqrt{\left(\rho_{11}-\rho_{44}\right)^{2}+4\left|\rho_{14}\right|^{2}}\right] \\
& \lambda_{3,4}=\frac{1}{2}\left[\rho_{22}+\rho_{33} \pm \sqrt{\left(\rho_{22}-\rho_{33}\right)^{2}+4\left|\rho_{23}\right|^{2}}\right] .
\end{aligned}
$$

Then, the quantum mutual information can be expressed as

$$
\mathcal{I}(\rho(t))=S\left(\rho^{(1)}(t)\right)+S\left(\rho^{(2)}(t)\right)+\sum_{i=1}^{4} \lambda_{i} \log _{2} \lambda_{i}
$$

where

$$
\begin{aligned}
S\left(\rho^{(1)}(t)\right)= & -\left(\rho_{11}+\rho_{22}\right) \log _{2}\left(\rho_{11}+\rho_{22}\right) \\
& -\left(\rho_{33}+\rho_{44}\right) \log _{2}\left(\rho_{33}+\rho_{44}\right), \\
S\left(\rho^{(2)}(t)\right)= & -\left(\rho_{11}+\rho_{33}\right) \log _{2}\left(\rho_{11}+\rho_{33}\right) \\
& -\left(\rho_{22}+\rho_{44}\right) \log _{2}\left(\rho_{22}+\rho_{44}\right),
\end{aligned}
$$

where $\rho^{(i)}(t)$ is the reduced density matrix of the $i$ th partite of the qubit-data bus system. By substituting $\rho_{i j}(i, j=1,2,3,4)$ of eq. (16) into eqs. (18)-(22), the explicit expressions of quantum discord $\mathcal{D}(\rho(t))$ and the quantum mutual information $\mathcal{I}(\rho(t))$ can be obtained [34]. Here we do not list the concrete expressions of the quantum discord and quantum mutual information because it is rather long and complex.

In order to quantify the entanglement dynamics of the qubit-data bus system and make a comparison with the quantum discord dynamics, we adopt the measure concurrence which is defined by [35]

$$
E=\max \left\{0, \sqrt{\eta_{1}}-\sqrt{\eta_{2}}-\sqrt{\eta_{3}}-\sqrt{\eta_{4}}\right\},
$$

where the $\eta_{i}(i=1,2,3,4)$ are the eigenvalues in decreasing order of the magnitude of the "spin-flipped" density matrix operator $R=\rho\left(\sigma_{y} \otimes \sigma_{y}\right) \rho^{*}\left(\sigma_{y} \otimes \sigma_{y}\right)$ and $\sigma_{y}$ is the Pauli $Y$ matrix, i.e., $\sigma_{y}=\left(\begin{array}{cc}0 & -i \\ i & 0\end{array}\right)$. Combining the definition of

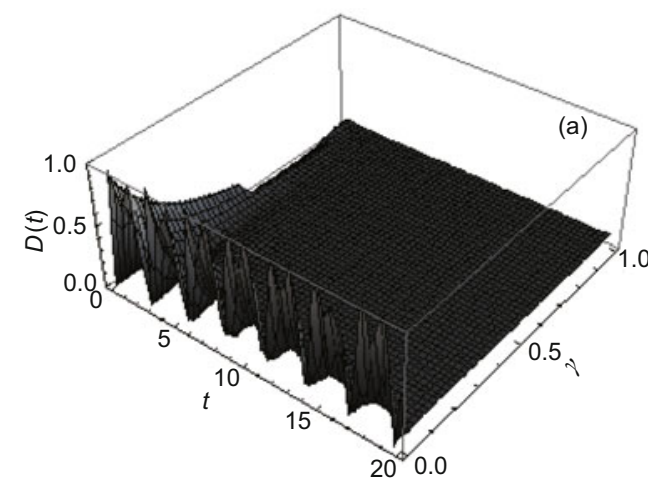

concurrence with the density matrix $\rho(t)$ of eq. (16), we can find the the explicit expressions of the concurrence between the superconducting qubit and data bus:

$$
E(t)=2 \max \left(0,\left|\rho_{32}\right|\right) \text {. }
$$

We plot the quantum discord (a) and entanglement (b) between the superconducting qubit and data bus in Figure 1 as a function of the evolution time $t$ and the decoherence coefficient $\gamma$ with $\chi=1, \omega=1, \omega_{q}=2, \omega_{e}=0, \lambda=0, n=0$. It can be seen from Figure 1 that the quantum discord and entanglement are always larger than zero during the time evolution if $\gamma \neq 0$ and $\omega \neq \omega_{q}$, which means that the phase decoherence does not completely destroy the quantum discord and entanglement but a stationary quantum discord and entanglement can arise in the interaction of the superconducting qubit and data bus for long-time evolution.

In Figure 2, the quantum discord (a) and entanglement (b) between the superconducting qubit and data bus are plotted as a function of the evolution time $t$ for two different values of $\omega_{q}: \omega_{q}=1$ (solid line), $\omega_{q}=2$ (dashed line) with $\chi=1$, $\omega=1, \omega_{e}=\lambda=0, \gamma=0.05, n=0$. We can see from Figure 2 that the quantum discord and entanglement between the superconducting qubit and data bus oscillate and eventually become zero when $\omega=\omega_{q}$ in the presence of phase decoherence. But the quantum discord and entanglement between the superconducting qubit and data bus are not completely destroyed and remain at a stationary non-zero value in the case of $\omega \neq \omega_{q}$ when the time approaches infinite.

In order to study the influence of TDEF on the dynamics of quantum discord and entanglement between the superconducting qubit and data bus, we plot the discord (a) and entanglement (b) between the superconducting qubit and data bus in Figure 3 as a function of the evolution time $t$ for different values of $\omega_{e}$ and $\lambda$ : $\omega_{e}=\lambda=0$ (solid line), $\omega_{e}=1, \lambda=0.65$ (dashed line) with $\chi=1, \omega=1, \omega_{q}=2, \gamma=0.05, n=0$. By comparing the solid line and dashed line in Figure 3(a) and (b), we can see clearly that the amount of stationary quantum

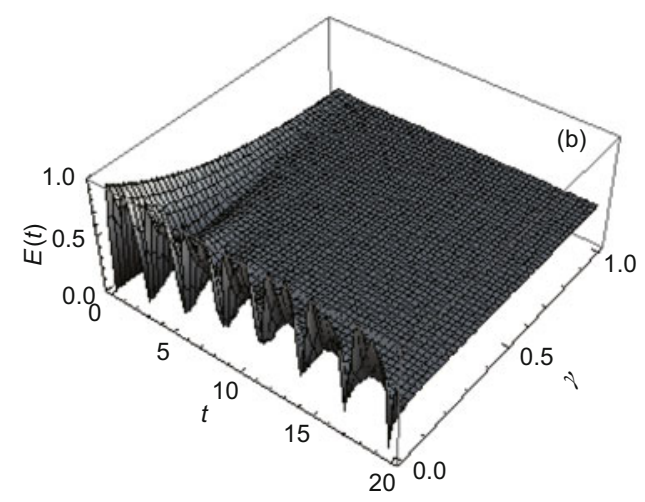

Figure 1 The quantum discord (a) and entanglement (b) between the superconducting qubit and data bus are plotted as a function of the evolution time $t$ and the decoherence coefficient $\gamma$ with $\chi=1, \omega=1, \omega_{q}=2, \omega_{e}=0, \lambda=0, n=0$. 

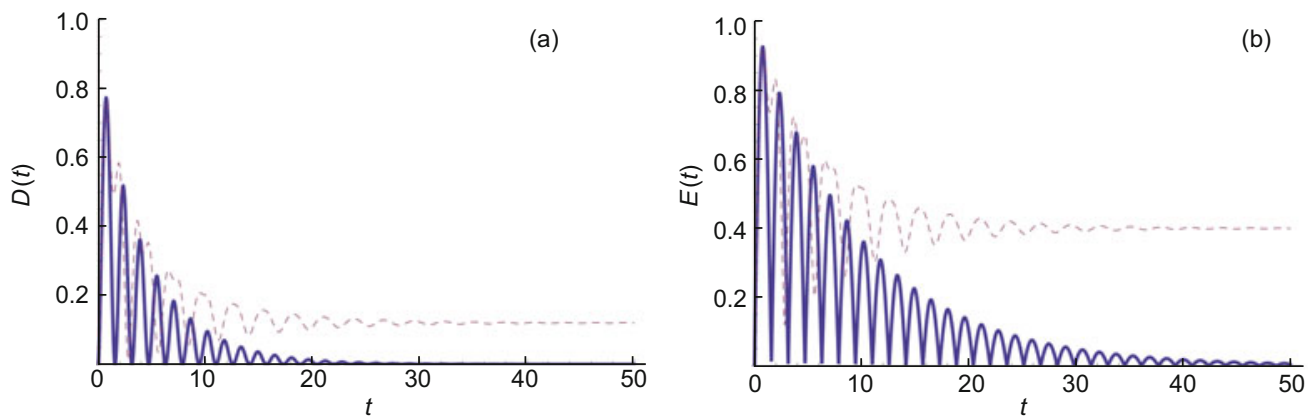

(b)

Figure 2 (Color online) The quantum discord (a) and entanglement (b) between the superconducting qubit and data bus are plotted as a function of the evolution time $t$ for different values of $\omega_{q}: \omega_{q}=1$ (solid line), $\omega_{q}=2$ (dashed line) with $\chi=1, \omega=1, \omega_{e}=\lambda=0, \gamma=0.05, n=0$.
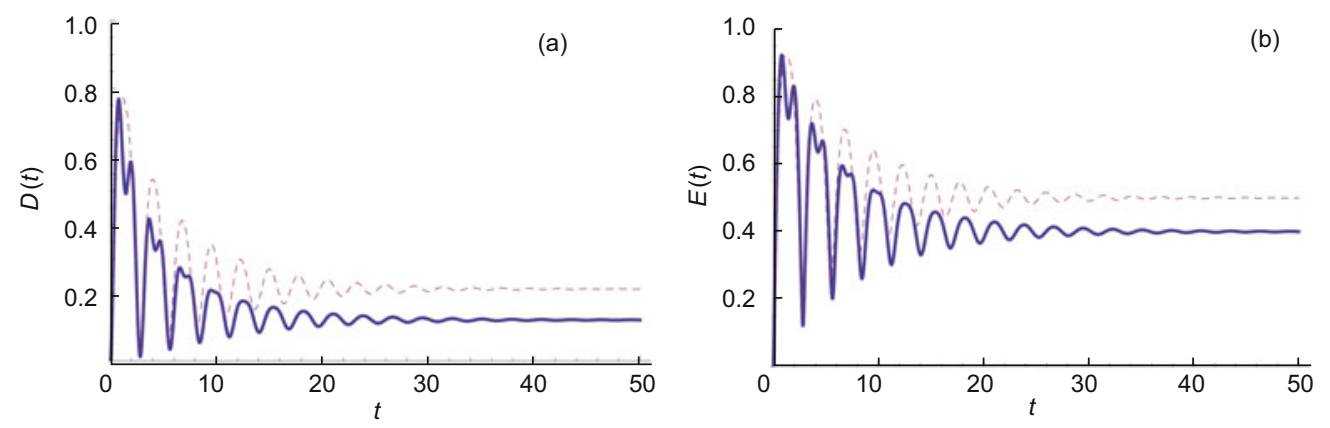

Figure 3 (Color online) The quantum discord (a) and entanglement (b) between the superconducting qubit and data bus are plotted as a function of the evolution time $t$ for different values of $\omega_{e}$ and $\lambda: \omega_{e}=\lambda=0$ (solid line), $\omega_{e}=1, \lambda=0.65$ (dashed line) with $\chi=1, \omega=1, \omega_{q}=2, \gamma=0.05, n=0$.

discord and entanglement between the superconducting qubit and data bus can be increased by adjusting the frequency $\omega_{e}$ and strength $\lambda$ of the TDEF in the presence of phase decoherence.

Finally, the influence of the initial state of data bus on the dynamics of quantum discord and entanglement between the superconducting qubit and data bus are studied as the quantum discord (a) and entanglement (b) are plotted as a function of the evolution time $t$ in Figure 4 for different values of $n: n=0$ (solid line), $n=1$ (dashed line) with $\chi=1, \omega=1, \omega_{q}=2, \omega_{e}=\lambda=0, \gamma=0.05$. It is found that the oscillation of quantum discord and entanglement end earlier and the stationary amount of quantum discord and en- tanglement become smaller as $n$ increases. Therefore, in order to generate optimal amount of stationary quantum discord and stationary entanglement between the qubit and data bus, it would be best the initial state of data bus is prepared in $|0\rangle$.

\section{Conclusions}

In this paper, we investigate the dynamics of quantum discord and entanglement between a superconducting qubit and a data bus, which is driven by a controllable TDEF, in the presence of phase decoherence. It is found that the quantum discord and entanglement between the superconducting
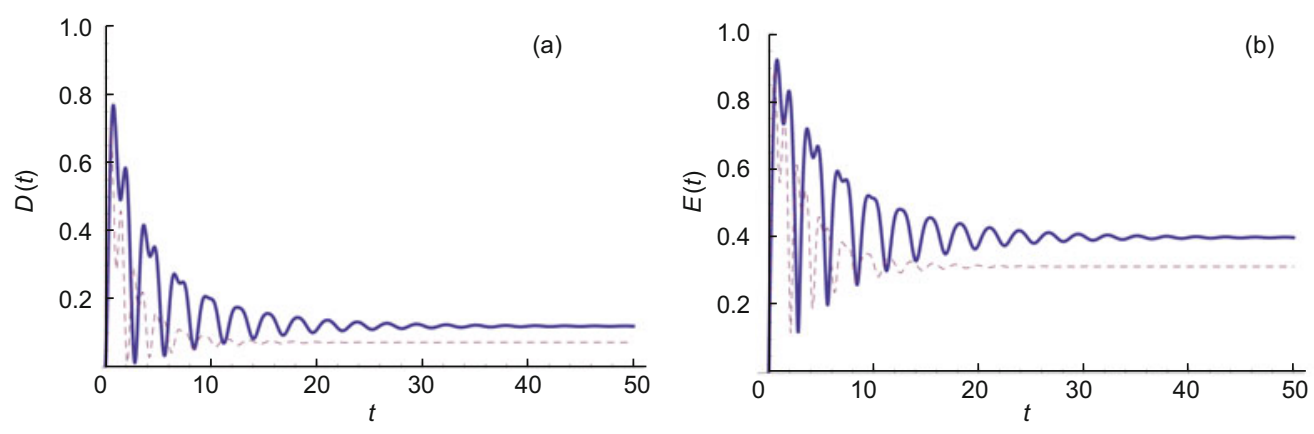

Figure 4 (Color online) The quantum discord (a) and entanglement (b) between the superconducting qubit and data bus are plotted as a function of the evolution time $t$ for different values of $n: n=0$ (solid line), $n=1$ (dashed line) with $\chi=1, \omega=1, \omega_{q}=2, \omega_{e}=\lambda=0, \gamma=0.05$. 
qubit and data bus rapidly decay with phase decoherence in the case of $\omega=\omega_{q}$. However, in the case of $\omega \neq \omega_{q}$, the quantum discord and entanglement between the superconducting qubit and data bus are not completely destroyed by the phase decoherence, which means that a stationary quantum discord and entanglement can arise in the interaction of the superconducting qubit with data bus as the time approach to infinite. We also examine the influence of the TDEF on the dynamics of quantum discord and entanglement between the superconducting qubit and data bus and show that the amount of stationary quantum discord and entanglement can be increased by applying the TDEF. Finally we study the influence of the initial state of data bus on the dynamics of quantum discord and entanglement between the superconducting qubit and data bus and show that the oscillation of quantum discord and entanglement end earlier and the stationary amount of quantum discord and entanglement become smaller as $n$ increases. The approach adopted here may be extended to improve the implementation of quantum tasks which based on the quantum correlations.

1 Nielsen M A, Chuand I L. Quantum Computation and Quantum Information. Cambridge: Cambridge University Press, 2000

2 Hagley E, Maitre E, Nogues G, et al. Generation of Einstein-PodolskyRosen pairs of atoms. Phys Rev Lett, 1997, 79: 1-5

3 Rauschenbeutel A, Nogues G, Osnaghi S, et al. Step-by-step engineered multiparticle entanglement. Science, 2000, 288: 2024-2028

4 Brennecke F, Donner T, Ritter S, et al. Cavity QED with a Bose-Einstein condensate. Nature, 2007, 450: 268-271

5 McKeever J, Buck J R, Boozer A D, et al. State-insensitive cooling and trapping of single atoms in an optical cavity. Phys Rev Lett, 2003, 90: 133602

6 Deng Z J, Feng M, Gao K L. Preparation of entangled states of four remote atomic qubits in decoherence-free subspace. Phys Rev A, 2007, 75: 024302

7 Braunstein S L, Caves C M, Jozsa R, et al. Separability of very noisy mixed states and implications for NMR quantum computing. Phys Rev Lett, 1999, 83: 1054-1057

8 Meyer D A. Sophisticated quantum search without entanglement. Phys Rev Lett, 2000, 85: 2014-2017

9 Bennett C H, DiVincenzo D P, Fuchs C A, et al. Quantum nonlocality without entanglement. Phys Rev A, 1999, 59: 1070-1091

10 Datta A, Shaji A, Caves C M. Quantum discord and the power of one qubit. Phys Rev Lett, 2008, 100: 050502

11 Lanyon B P, Barbieri M, Almeida M P, et al. Experimental quantum computing without entanglement. Phys Rev Lett, 2008, 101: 200501

12 Ollivier H, Zurek W H. Quantum discord: A measure of the quantumness of correlations. Phys Rev Lett, 2001, 88: 017901
13 Horodecki M, Horodecki P, Horodecki R, et al. Local versus nonlocal information in quantum-information theory: Formalism and phenomena. Phys Rev A, 2005, 71: 062307

14 Vedral V. The elusive source of quantum speedup. Found Phys, 2010, 40: 1141-1154

15 Werlang T, Souza S, Fanchini F F, et al. Robustness of quantum discord to sudden death. Phys Rev A, 2009, 80: 024103

16 Salemian S, Mohammadnejad S. An error-free protocol for quantum entanglement distribution in long-distance quantum communication. Chin Sci Bull, 2011, 56: 618-625

17 Chen C Y, Sun Q. Realization of superposition and entanglement of coherent and squeezed states in circuit quantum electrodynamics. Sci China Phys Mech Astron, 2011, 54: 930-935

18 Cao H X, Li L, Chen Z L. Restricted allowable generalized quantum gates. Chin Sci Bull, 2010, 55: 2122-2125

19 Dong H, Liu X F, Sun C P. Thermodynamic witness of quantum probing. Chin Sci Bull, 2010, 55: 3256-3260

20 Wang B, Xu Z Y, Chen Z Q, et al. Non-Markovian effect on the quantum discord. Phys Rev A, 2010, 81: 014101

21 Hao X, Ma C L, Sha J Q. Decoherence of quantum discord in an asymmetric-anisotropy spin system. J Phys A: Math Theor, 2010, 43: 425302

22 Mazzola L, Piilo J, Maniscalco S. Sudden transition between classical and quantum decoherence. Phys Rev Lett, 2010, 104: 200401

$23 \mathrm{Xu} \mathrm{J} \mathrm{S,} \mathrm{Xu} \mathrm{X} \mathrm{Y,} \mathrm{Li} \mathrm{C} \mathrm{F,} \mathrm{et} \mathrm{al.} \mathrm{Experimental} \mathrm{investigation} \mathrm{of} \mathrm{classical}$ and quantum correlations under decoherence. Nat Commun, 2010, 1: 7

24 Makhlin Y, Schön G, Snirman A. Quantum-state engineering with Josephson-junction devices. Rev Mod Phys, 2001, 73: 357-400

25 Clarke J, Wilhelm F K. Superconducting quantum bits. Nature, 2008, 453: 1031-1042

26 Majer J, Chow J M, Gambetta J M, et al. Coupling superconducting qubits via a cavity bus. Nature, 2007, 449: 443-447

27 You J Q, Tsai J S, Nori F. Scalable quantum computing with Josephson charge qubits. Phys Rev Lett, 2002, 89: 197902

28 Zagoskin A M, Grajcar M, Omelyanchouk A N. Selective amplification of a quantum state. Phys Rev A, 2004, 70: 060301

29 Blais A, van den Brink A M, Zagoskin A M. Tunable coupling of auperconducting qubits. Phys Rev Lett, 2003, 90: 127901

30 Liu Y X, Sun C P, Nori F. Scalable superconducting qubit circuits using dressed states. Phys Rev A, 2006, 74: 052321

31 Liu Y X, You J Q, Wei L F, et al. Optical selection rules and phasedependent adiabatic state control in a superconducting quantum circuit. Phys Rev Lett, 2005, 95: 087001

$32 \mathrm{Xu} \mathrm{J} \mathrm{B,} \mathrm{Li} \mathrm{S} \mathrm{B.} \mathrm{Entanglement} \mathrm{and} \mathrm{Bell} \mathrm{violation} \mathrm{with} \mathrm{phase} \mathrm{decoherence}$ or dissipation. Eur Phys J D, 2005, 35: 553-560

33 Xu J B, Zhang Y Q. Entanglement control in a superconducting qubit system by an electromagnetic field. Eur Phys J D, 2011, 63: 483-488

34 Ali M, Rau A R P, Alber G. Quantum discord for two-qubit X states. Phys Rev A, 2010, 81: 042105

35 Wootters W K. Entanglement of formation of an arbitrary state of two qubits. Phys Rev Lett, 1998, 80: 2245-2248

Open Access This article is distributed under the terms of the Creative Commons Attribution License which permits any use, distribution, and reproduction in any medium, provided the original author(s) and source are credited. 